\title{
The effects of slow-paced versus mechanically assisted breathing on autonomic function in fibromyalgia patients
}

This article was published in the following Dove Press journal: Journal of Pain Research

\author{
John E Schmidt' \\ Travis G O'Brien² \\ W Michael Hooten ${ }^{3}$ \\ Michael J Joyner ${ }^{3}$ \\ Bruce D Johnson ${ }^{4,5}$ \\ 'Department of Psychology, \\ Navy Medicine Professional \\ Development Center, Walter Reed \\ National Military Medical Center, \\ Uniformed Services University of \\ the Health Sciences, Bethesda, MD, \\ ${ }^{2}$ Department of Physical Medicine \\ and Rehabilitation, ${ }^{3}$ Department of \\ Anesthesiology, Division of Pain \\ Medicine, ${ }^{4}$ Department of Medicine, \\ ${ }^{5}$ Department of Physiology, Mayo \\ Clinic, Rochester, MN, USA
}

Correspondence: John E Schmidt Department of Psychology, Navy Medicine Professional Development Center, Walter Reed National Military Medical Center, Uniformed Services University of the Health Sciences, 8955 Wood Road, Bethesda, MD 20889-5628, USA

Tel + I 30I 3198193

Email John.e.schmidt.civ@mail.mil

\begin{abstract}
Paced breathing has shown efficacy in fibromyalgia (FM), but the mechanisms associated with symptom change are largely unknown. We investigated whether changes in respiratory rate (RR) alone resulted in autonomic changes during normal, paced, and mechanically assisted breathing in untrained FM patients and controls. Participants included 20 FM patients and 14 controls matched for age and body mass index. During a single visit, participants completed three 15-minute breathing sessions: 1) normal breathing, 2) slow-paced breathing, and 3) mechanically assisted breathing (continuous positive airway pressure) while supine. Continuous blood pressure and electrocardiogram were recorded, and measures of heart rate variability (HRV) and spontaneous baroreceptor sensitivity (sBRS) were calculated. During normal breathing, FM patients had higher heart rate (HR), but lower HRV and sBRS variables compared to controls with no difference in RR. Compared to the paced breathing condition, FM patients had significantly lower HR with higher HRV and sBRS variables during mechanically assisted breathing, despite no significant change in RR. Mechanically assisted breathing provided greater benefits in autonomic function than paced breathing in untrained FM patients. Future research will be needed to elucidate the central pathways involved in these autonomic changes and whether training in paced breathing can eventually replicate the results seen in mechanically assisted patients.
\end{abstract}

Keywords: fibromyalgia, heart rate variability, baroreceptor sensitivity, paced breathing, autonomic activity, mechanically assisted breathing

\section{Introduction}

Fibromyalgia (FM) is a disorder characterized by chronic widespread pain. ${ }^{11,12}$ Individuals diagnosed with FM often report significant symptoms of comorbid fatigue, sleep dysfunction, and limited physical activity. ${ }^{4,10}$ Psychologically, they frequently present with symptoms of depression, anxiety, and catastrophizing about the impact pain has on daily routine, work, and social activities. ${ }^{2,14}$ FM is typically assessed and treated within the biopsychosocial model framework, and multidisciplinary interventions that include medications and behavioral pain management strategies have demonstrated the most improvement in pain severity and daily functioning. ${ }^{3}$ Several recently published studies have demonstrated improvements in pain and other FM-related symptoms in patients after training in a breathing-focused intervention. ${ }^{18,37,38}$ Specifically, heart rate variability (HRV) biofeedback paired with slow-paced breathing has demonstrated efficacy in the improvement of pain severity and functioning in FM. ${ }^{18}$ However, the underlying physiological changes associated with the efficacy of these interventions are not well understood. 
Physiologic dynamics among cardiac, respiratory, and blood pressure regulation in humans have been explored under various conditions, including normal breathing, paced breathing, yogic breathing, and Zen Ranzai practice. ${ }^{17,19,34}$ In healthy subjects, slow-paced breathing increases the amplitude of respiratory sinus arrhythmia (RSA), which refers to the cyclical fluctuations in heart rate (HR) that correlate with respiratory patterns. RSA is assessed using high-frequency $\mathrm{HRV}$, is vagally mediated, and is used as a noninvasive measure of parasympathetic function. ${ }^{5,6}$ Respiratory rate (RR) also stimulates carotid baroreceptors through oscillatory changes in stroke volume and arterial blood pressure. Spontaneous baroreceptor sensitivity (sBRS) is a measure of the increase in the interval between heartbeats per $\mathrm{mmHg}$ arterial blood pressure rise. Lehrer et al have explored the interactions among HRV, respiratory activity, and baroreflexes through an HRV biofeedback protocol. ${ }^{25,26}$. At a personally unique frequency, HR and respiration are in phase and result in higher RSA through the interaction of respiratory activity and baroreflex response. However, these studies reported on patients who were trained in slow-paced breathing or HRV biofeedback. The primary aim of this study was to explore whether different breathing conditions were associated with changes in HRV and sBRS in untrained FM patients. Study participants were assessed during normal, slow-paced, and mechanically assisted breathing conditions. We hypothesized that similar improvements in autonomic function would be observed in both the slow-paced and mechanically assisted breathing conditions if RR was the same.

\section{Patients and methods}

\section{Participants}

Study procedures were approved by the Mayo Clinic Institutional Review Board, and all participants provided written informed consent. Participants were recruited from the Mayo Clinic Department of Medicine Fibromyalgia Treatment Program or from the local community (matched healthy controls). Inclusion criteria for the participants with FM were as follows: 1 ) aged $\geq 18$ years; 2) female; 3) clinical diagnosis of FM per the American College of Rheumatology 2011 diagnostic criteria; ${ }^{46} 4$ ) pain duration of at least 6 months; 5) moderate pain level defined by at least 30 on a 0-100 mm visual analog scale (VAS). ${ }^{13}$ Exclusion criteria for both patients and controls included: 1) past or current history of hypertension or heart disease; 2) current use of narcotic medications; 3) history of asthma or other chronic respiratory conditions; 4) history of diabetes; 5) pregnant at the time of study participation assessed via urinalysis; 6) systolic blood pressure $(\mathrm{SBP})>140 \mathrm{mmHg}$ or diastolic blood pressure $>90$ mmHg. ${ }^{9}$ Participants taking non-narcotic medications were maintained on their prescribed schedule while participating in the study. Exclusion criteria were determined by self-report and medical record review during initial screening. Healthy controls were matched to the FM participants on age and body mass index (BMI). Potential control participants were excluded if they reported pain of $>0$ on a $0-10$ pain VAS or had any history of chronic pain conditions.

\section{Current stage of menstrual cycle}

During study screening, day of menstrual cycle was recorded by asking for the last day of the previous menstrual period. Research has demonstrated that autonomic regulation of the heart fluctuates during the menstrual cycle with HRV being lower in the luteal phase (days 15-28) than in other phases. ${ }^{23,36}$ The results of these studies suggest that sympathetic nervous system activity is dominant during the luteal phase, possibly due to higher concentrations of progesterone. All participants were studied in the early follicular phase of the menstrual cycle or in the low hormone phase of oral contraceptive use.

\section{Procedures}

\section{Study conditions and physiologic assessment}

The laboratory assessment was completed in one visit ( $~ 90$ minutes). Study participants completed the self-report measures (described below) and were then instrumented and tested in the supine position. Instrumentation was followed by a 5 -minute acclimation period for the participant to adjust to the laboratory environment and to ensure equipment was functioning correctly. Following the acclimation period, the participants were given instructions for three breathing conditions, each lasting 15 minutes with a 5-minute rest period between each condition. The first condition was normal breathing with the following instruction: "For the next 15 minutes, I would like you to breathe normally and rest quietly." The second breathing condition was slow-paced breathing with the following instruction: "For the next 15 minutes, I would like you to breathe with the visual metronome, inhaling as the bar goes up, and exhaling as the bar goes down." The slow-paced breathing visual metronome was set for an inhalation period of 5 seconds and an exhalation period of 5 seconds and was running on a laptop set up for easy viewing by the participant. The final condition was assisted breathing using a C-PAP machine with the following instruction: "For the next 15 minutes, I would like you to breathe with the mechanical ventilator." The C-PAP machine 
was set for a respiration rate of six breaths per minute to match the slow-paced breathing condition.

The physiologic assessment configuration for this study included the measurement of electrocardiogram (ECG), continuous blood pressure, and RR. ECG was recorded using 3-leads (Cardiocap) placed in the Lead II configuration. Continuous blood pressure was recorded on a beat-to-beat basis by finger photoplethysmography (Finometer) regularly verified by automated sphygmomanometry on the contralateral arm. RR was recorded using thoracic and diaphragmatic belted strain-gauges (Respiratrace). All physiologic measures were recorded using the WINDAQ data acquisition system at a sampling rate of $500 \mathrm{~Hz}$.

\section{Standardized psychologic assessment Pain VAS}

The VAS is a $100-\mathrm{mm}$ line with the endpoints anchored by "0: no pain" and "100: worst pain imaginable." The participants were instructed to place a mark on the line indicating the present pain level.

\section{Center for Epidemiologic Studies Depression Scale (CES-D)}

The CES-D is a 20-item measure of the presence and severity of depressive symptoms. ${ }^{32} \mathrm{~A}$ cutoff score of $\geq 16$ is associated with depressed outpatients. ${ }^{45}$ Test-retest reliability, internal consistency, and convergent validity are adequate. ${ }^{33}$

\section{State-Trait Anxiety Inventory (STAI)}

The STAI is a 40-item measure used to assess individual differences related to state and trait anxiety constructs. ${ }^{41}$ Scores on this scale range from 20 to 80 , and higher scores indicate greater anxiety. The STAI has consistently demonstrated good psychometric properties in patients with chronic pain. ${ }^{28}$

\section{Pittsburgh Sleep Quality Index (PSQI)}

The PSQI is a 12-item comprehensive measure of sleep quality. It has exhibited test-retest stability (full scale $r=0.85$ ) and good overall internal consistency $(\alpha=0.83)$ and provides a valid and reliable assessment of overall sleep quality and disturbance. ${ }^{8}$

\section{Multidimensional Fatigue Symptom Inventory (MFSI)}

The MFSI is a 30-item measure designed to identify five facets of fatigue: global experience, somatic symptoms, cognitive symptoms, affective symptoms, and behavioral symptoms. ${ }^{43}$ Participants rate each statement according to how true it has been for them over the past 7 days.

\section{Autonomic activity}

HRV was used as a measure of autonomic activity. To calculate HRV, the ECG signal was filtered and transformed into normal-to-normal (NN) intervals using the Windaq Waveform Browser software. These data were imported into the Nevrokard Advanced HRV analysis software, version 10.1.0, for time domain analyses (Nevrokard Kiauta, k.d., Izola, Slovenia). For this study, the time domain measure of root mean square of successive differences of NN intervals (RMSSD) is reported.

sBRS index was also calculated and reported. The time domain sBRS index is based on the quantification of sequences of three heart beats in which SBP consecutively increases or decreases by at least $0.5 \mathrm{mmHg}$, accompanied by changes in the same direction of the NN intervals by at least $5 \mathrm{~ms}$ of the subsequent beats. ${ }^{7]}$ To complete the time domain sBRS, ECG and continuous blood pressure data were imported into the Nevrokard Baroreflex Sensitivity Analysis software, version 5.7.0.

\section{Data analysis}

Data were analyzed using the SPSS statistical package (version 21.0; IBM Corporation, Armonk, NY, USA). Descriptive statistics included mean and standard deviations for all demographic, questionnaire, and physiological data. Any outlying scores were compared to the original data to ensure there were no data entry errors. The alpha level for all analyses was set at $p<0.05$. The study hypotheses were evaluated by using repeated measures analysis of variance (ANOVA) for within group comparisons. Study groups were compared on self-report and normal breathing physiological measures using ANOVA. Means and standard deviations of the physiological data for each full 15-minute recording period are reported for HR, mean arterial pressure (MAP), and RR. The HRV and sBRS values were calculated using the Nevrokard software using data from each 15-minute recording period.

\section{Results Demographics}

Study participants were patients with a diagnosis of FM $(n=20)$ and healthy controls $(n=14)$ matched on age (mean age FM group $=46.2$ [SD =9.0] vs control group $=47.0$ [SD $=9.0], p=0.801$ ) and BMI (mean BMI FM group $=28.3$ [SD $=8.5]$ vs control group $=26.8$ [SD =4.7], $p=0.535$ ). The FM group patients reported a mean present VAS pain score of $31.8 \mathrm{~mm}(\mathrm{SD}=16.9)$ and a mean average VAS score over the past week of $50.3 \mathrm{~mm}(\mathrm{SD}=18.9)$. Control group participants did not report any pain at the start of laboratory assessment. 


\section{Affect and physical functioning}

The FM group reported significantly higher rates of depression (CES-D; $F=35.32, p<0.001$ ), trait and state anxiety (STAI; trait $F=18.97, p<0.001$; state $F=9.91, p=0.004$ ), sleep dysfunction (PSQI; $F=28.42, p<0.001$ ), and fatigue (MFSI total score; $F=56.76, p<0.001)$ compared to the control group. Table 1 summarizes the details on affect and physical functioning measures.

\section{Normal breathing physiologic variables}

During normal breathing, the FM group had significantly higher HR $(F=6.93, p=0.014)$, but significantly lower $\operatorname{RMSSD}(F=7.43, p=0.011)$ and sBRS $(F=7.28, p=0.012)$ compared to the control group. No significant differences between the two groups were found on MAP and RR. Table 2 provides details regarding physiologic data during normal breathing.

\section{Change in physiologic variables}

When compared across study conditions, the FM group had significant change in HR $(F=3.35, p=0.048)$, RMSSD $(F=16.03, p<0.001)$ and $\operatorname{sBRS}(F=5.671, p=0.010)$. Pairwise comparisons across the three study conditions on these variables showed significant differences between the mechanically assisted breathing condition and the other

Table I Psychological and functional measures at baseline

\begin{tabular}{cllll}
\hline Variables & $\begin{array}{l}\text { FM, mean } \\
(\mathbf{S D})\end{array}$ & $\begin{array}{l}\text { Control, } \\
\text { mean (SD) }\end{array}$ & $\boldsymbol{F}$ & p-value \\
\hline CES-D & 19.7 & 3.4 & 35.3 & 0.000 \\
& $(8.7)$ & $(5.9)$ & & \\
STAI-Trait & 43.5 & 26.0 & 19.0 & 0.000 \\
& $(12.6)$ & $(5.5)$ & & \\
STAI-State & 39.4 & 25.8 & 9.91 & 0.004 \\
& $(13.3)$ & $(7.0)$ & & \\
PSQI & 11.0 & 4.2 & 28.4 & 0.000 \\
& $(3.8)$ & $(2.9)$ & & \\
MFSI & 39.4 & -9.9 & 56.8 & 0.000 \\
(total score) & $(19.4)$ & $(16.4)$ & & \\
General & 16.7 & 3.5 & 39.9 & 0.000 \\
& $(5.4)$ & $(6.4)$ & & \\
Emotional & 7.8 & 1.0 & 19.5 & 0.000 \\
& $(5.3)$ & $(1.9)$ & & \\
Physical & 12.5 & 1.3 & 42.2 & 0.000 \\
& $(5.8)$ & $(2.7)$ & & \\
Mental & 11.5 & 1.8 & 38.5 & 0.000 \\
& $(5.3)$ & $(2.3)$ & & \\
Vigor & 9.0 & 17.5 & 29.8 & 0.000 \\
& $(4.2)$ & $(4.5)$ & & \\
\hline
\end{tabular}

Abbreviations: FM, fibromyalgia; SD, standard deviation; CES-D, Center for Epidemiological Studies Depression measure; STAI, State Trait Anxiety Inventory; PSQI, Pittsburgh Sleep Quality Index; MFSI, Multidimensional Fatigue Symptom Inventory. two conditions (all $p$ 's $<0.05$ ). When compared across study conditions, no significant changes in physiological variables were detected in the control group. Note that both the groups had a significant change in RR as expected. Tables 3 and 4 provide details regarding within-group comparisons among study conditions. Figures 1 and 2 graphically display the sBRS and RMSSD values by condition for each group. Post hoc comparisons show where the two groups are significantly different on these variables.

\section{Discussion}

This physiologic study provides insight into the autonomic changes that occur with controlled breathing techniques in patients with FM. Slow-paced breathing, the practice of slow

Table 2 Physiologic measures between study groups during normal breathing

\begin{tabular}{lllll}
\hline Variables & $\begin{array}{l}\text { FM, mean } \\
\text { (SD) }\end{array}$ & $\begin{array}{l}\text { Control, } \\
\text { mean (SD) }\end{array}$ & F & p-value \\
\hline Heart rate (beats per & 73.2 & 64.0 & 6.93 & $0.014^{*}$ \\
minute) & $(10.4)$ & $(7.1)$ & & \\
MAP (mmHg) & 79.2 & 82.5 & 0.65 & 0.427 \\
& $(10.8)$ & $(10.5)$ & & \\
sBRS (ms/mmHg) & 8.9 & 14.8 & 7.28 & $0.012^{*}$ \\
& $(4.5)$ & $(7.5)$ & & \\
RMSSD (ms) & 25.2 & 42.9 & 7.43 & $0.011^{*}$ \\
& $(19.7)$ & $(19.7)$ & & \\
Respiratory rate & 14.7 & 12.8 & 2.69 & 0.112 \\
(breaths per minute) & $(3.7)$ & $(2.0)$ & & \\
\hline
\end{tabular}

Note: ${ }^{*} p<0.05$.

Abbreviations: FM, fibromyalgia; SD, standard deviation; MAP, mean arterial pressure; sBRS, spontaneous baroreceptor sensitivity; RMSSD, root mean square of the SD of normal-to-normal intervals.

Table 3 Repeated measures between experimental breathing conditions for the FM group

\begin{tabular}{|c|c|c|c|c|c|}
\hline \multirow[t]{2}{*}{ Variables } & \multicolumn{3}{|c|}{ Breathing condition, mean (SD) } & \multirow[t]{2}{*}{$\boldsymbol{F}$} & \multirow[t]{2}{*}{$p$-value } \\
\hline & Normal & $\begin{array}{l}\text { Slow- } \\
\text { paced }\end{array}$ & $\begin{array}{l}\text { Mechanically } \\
\text { assisted }\end{array}$ & & \\
\hline $\begin{array}{l}\text { Heart rate (beats } \\
\text { per minute) }\end{array}$ & $\begin{array}{l}72.92^{\mathrm{a}} \\
(10.7)\end{array}$ & $\begin{array}{l}72.18^{\mathrm{a}} \\
(12.7)\end{array}$ & $\begin{array}{l}69.62^{b} \\
(10.1)\end{array}$ & 3.35 & 0.048 \\
\hline MAP $(\mathrm{mmHg})$ & $\begin{array}{l}79.06 \\
(11.3)\end{array}$ & $\begin{array}{l}79.74 \\
(8.6)\end{array}$ & $\begin{array}{l}84.65 \\
(11.3)\end{array}$ & 2.86 & 0.077 \\
\hline $\begin{array}{l}\text { sBRS } \\
(\mathrm{ms} / \mathrm{mmHg})\end{array}$ & $\begin{array}{l}9.32^{\mathrm{a}} \\
(4.8)\end{array}$ & $\begin{array}{l}9.87^{\mathrm{a}} \\
(3.4)\end{array}$ & $\begin{array}{l}12.37^{b} \\
(4.2)\end{array}$ & 5.67 & 0.010 \\
\hline RMSS & $\begin{array}{l}21.48^{a} \\
(8.6)\end{array}$ & $\begin{array}{l}23.04^{\mathrm{a}} \\
(7.7)\end{array}$ & $\begin{array}{l}31.66^{\mathrm{b}} \\
(9.1)\end{array}$ & 16.03 & 0.000 \\
\hline $\begin{array}{l}\text { Respiratory rate } \\
\text { (breaths per } \\
\text { minute) }\end{array}$ & $\begin{array}{l}14.89^{\mathrm{a}} \\
(3.9)\end{array}$ & $\begin{array}{l}6.05^{b} \\
(1.7)\end{array}$ & $\begin{array}{l}7.50^{\mathrm{b}} \\
(2.8)\end{array}$ & 44.19 & 0.000 \\
\hline $\begin{array}{l}\text { Notes: }{ }^{a, b} \text { When sup } \\
\text { post hoc comparisor } \\
\text { When superscripts a } \\
\text { between group mean } \\
\text { Abbreviations: }\end{array}$ & $\begin{array}{l}n \text { indicates } \\
\text { ire differen } \\
\text { ns at } p<0.0\end{array}$ & gnif & $\begin{array}{l}\text { ween two cor } \\
\text { ifference bety } \\
\text { arison indicat }\end{array}$ & $\begin{array}{l}\text { ns on a } \\
\text { conditi } \\
\text { nificant }\end{array}$ & $\begin{array}{l}\text { a measure, } \\
\text { tion means. } \\
t \text { difference }\end{array}$ \\
\hline
\end{tabular}


timed breaths in response to anxiety-provoking stimuli, has been shown to alter autonomic nervous system (ANS) activity in multiple ways. In particular, previous studies have shown that sympathetic nervous system (SNS) activity decreases, as evidenced by changes in HR and blood pressure, while parasympathetic nervous system (PSNS) activity increases, as revealed by changes in HRV indices and sBRS. ${ }^{24,30,37,40}$ These physiological changes have been associated with a reduction in symptoms of chronic pain syndromes, including fear, anxiety, and catastrophizing. ${ }^{18,38,39,49}$ By understanding the mechanisms behind the therapeutic effects of slow-paced

Table 4 Repeated measures between experimental breathing conditions for the control group

\begin{tabular}{|c|c|c|c|c|c|}
\hline & \multicolumn{3}{|c|}{ Breathing condition, mean (SD) } & \multirow[t]{2}{*}{$\boldsymbol{F}$} & \multirow[t]{2}{*}{$p$-value } \\
\hline & Normal & $\begin{array}{l}\text { Slow- } \\
\text { paced }\end{array}$ & $\begin{array}{l}\text { Mechanically } \\
\text { assisted }\end{array}$ & & \\
\hline Heart rate (beats & 64.25 & 64.72 & 63.50 & 0.36 & 0.705 \\
\hline per minute) & $(8.1)$ & $(9.9)$ & $(9.4)$ & & \\
\hline \multirow[t]{2}{*}{ MAP $(\mathrm{mmHg})$} & 82.53 & 79.97 & 89.24 & 2.38 & 0.143 \\
\hline & $(6.7)$ & $(8.6)$ & $(10.2)$ & & \\
\hline $\mathrm{sBRS}(\mathrm{ms} /$ & 15.46 & 12.19 & 13.62 & 1.40 & 0.280 \\
\hline $\mathrm{mmHg})$ & $(9.1)$ & $(4.8)$ & $(7.1)$ & & \\
\hline \multirow[t]{2}{*}{ RMSSD (ms) } & 49.97 & 39.50 & 48.69 & 2.25 & 0.142 \\
\hline & $(19.6)$ & $(9.3)$ & $(13.9)$ & & \\
\hline Respiratory rate & $12.54^{\mathrm{a}}$ & $4.4 I^{b}$ & $5.45^{\mathrm{b}}$ & 74.30 & 0.000 \\
\hline (breaths per & $(1.0)$ & $(0.7)$ & $(1.0)$ & & \\
\hline minute) & & & & & \\
\hline \multicolumn{6}{|c|}{$\begin{array}{l}\text { Notes: }{ }^{a, b} \text { When superscripts are the same between two conditions on a measure, } \\
\text { post hoc comparison indicates no significant difference between condition means. } \\
\text { When superscripts are different, post hoc comparison indicates significant difference } \\
\text { between group means at } p<0.01 \text {. }\end{array}$} \\
\hline \multicolumn{6}{|c|}{$\begin{array}{l}\text { Abbreviations: SD, standard deviation; MAP, mean arterial pressure; sBRS, } \\
\text { spontaneous baroreceptor sensitivity; RMSSD, root mean square of the SD of } \\
\text { normal-to-normal intervals. }\end{array}$} \\
\hline
\end{tabular}

breathing, treatments can be refined and applied to other chronic pain conditions. Our work challenges the hypothesis that changing RR alone can drive the ANS changes responsible for the improvement in symptoms that occurs with slow-paced breathing.

In the present study during normal breathing, FM patients had measures of increased SNS activity and decreased PSNS activity compared to healthy pain-free controls. These results were anticipated, based on numerous prior studies characterizing the altered ANS activity in FM patients. ${ }^{20,22,29,44,47,48} \mathrm{HRV}$, the variation in time interval between heart beats, is generally determined by the balance of afferent input into the sinoatrial node. ${ }^{1,5}$ SNS activity leads to increases in HR but decreases in HRV. ${ }^{5}$ PSNS activity is responsible for most determinants of HRV, such as RSA, the change in NN interval between inspiration and expiration. ${ }^{48}$ PSNS input to baroreceptors in the carotid sinus and aortic arch increases their sensitivity to small changes in blood pressure, which increases HRV. ${ }^{26}$ Therefore, indices of HRV and sBRS activity have been used as reliable surrogates for PSNS activity in FM patients. ${ }^{5,6}$

We know that controlled breathing techniques can alter the maladaptive changes in ANS activity that exacerbate symptoms of chronic pain, but have little understanding of how this occurs. To clarify the role of RR on autonomic changes, we measured markers of ANS activity in FM and control patients under both slow-paced and mechanically assisted breathing conditions. When comparing FM patients between slow-paced and mechanically assisted breathing, HR decreased and measures of both HRV and sBRS increased in the mechanically assisted condition. These changes occurred

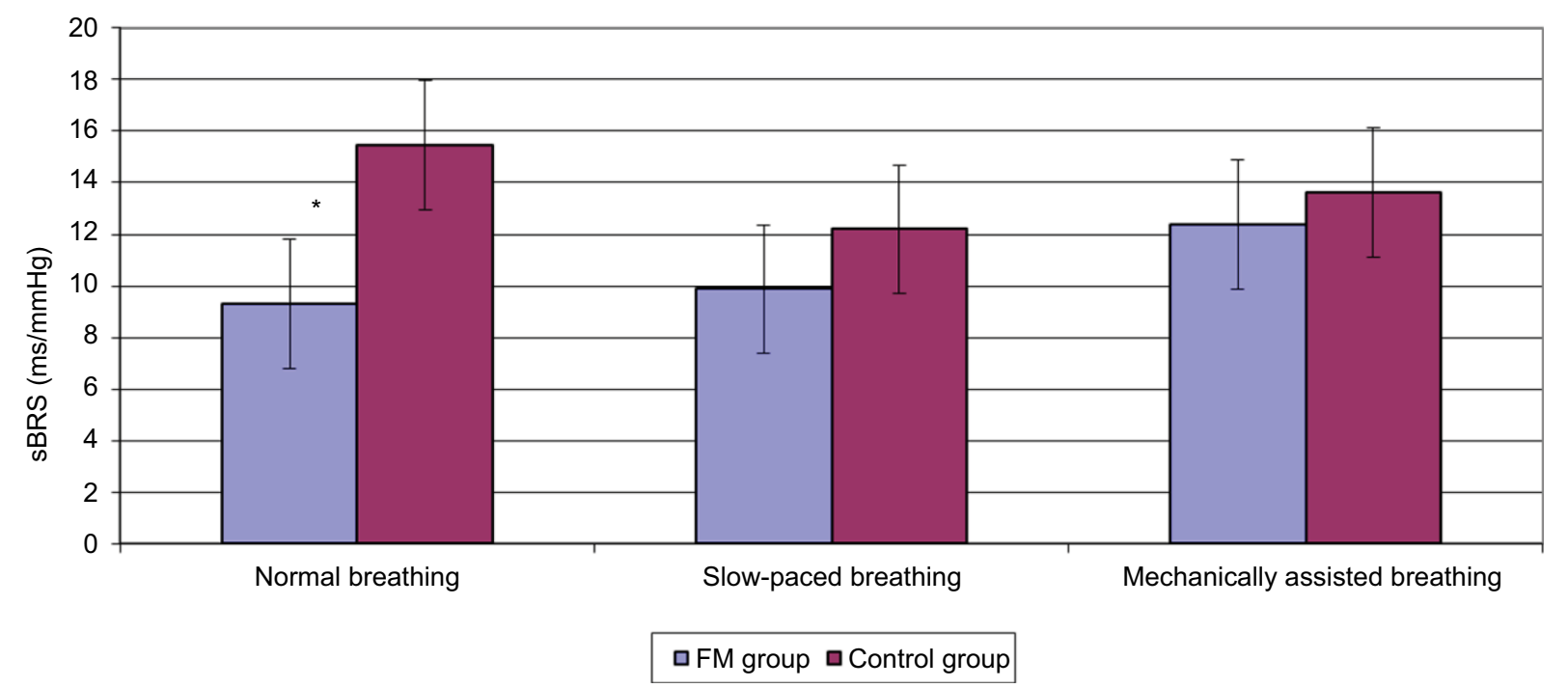

Figure I Baroreceptor function across conditions by group.

Note: $* p<0.05$.

Abbreviations: FM, fibromyalgia; sBRS, spontaneous baroreceptor sensitivity. 


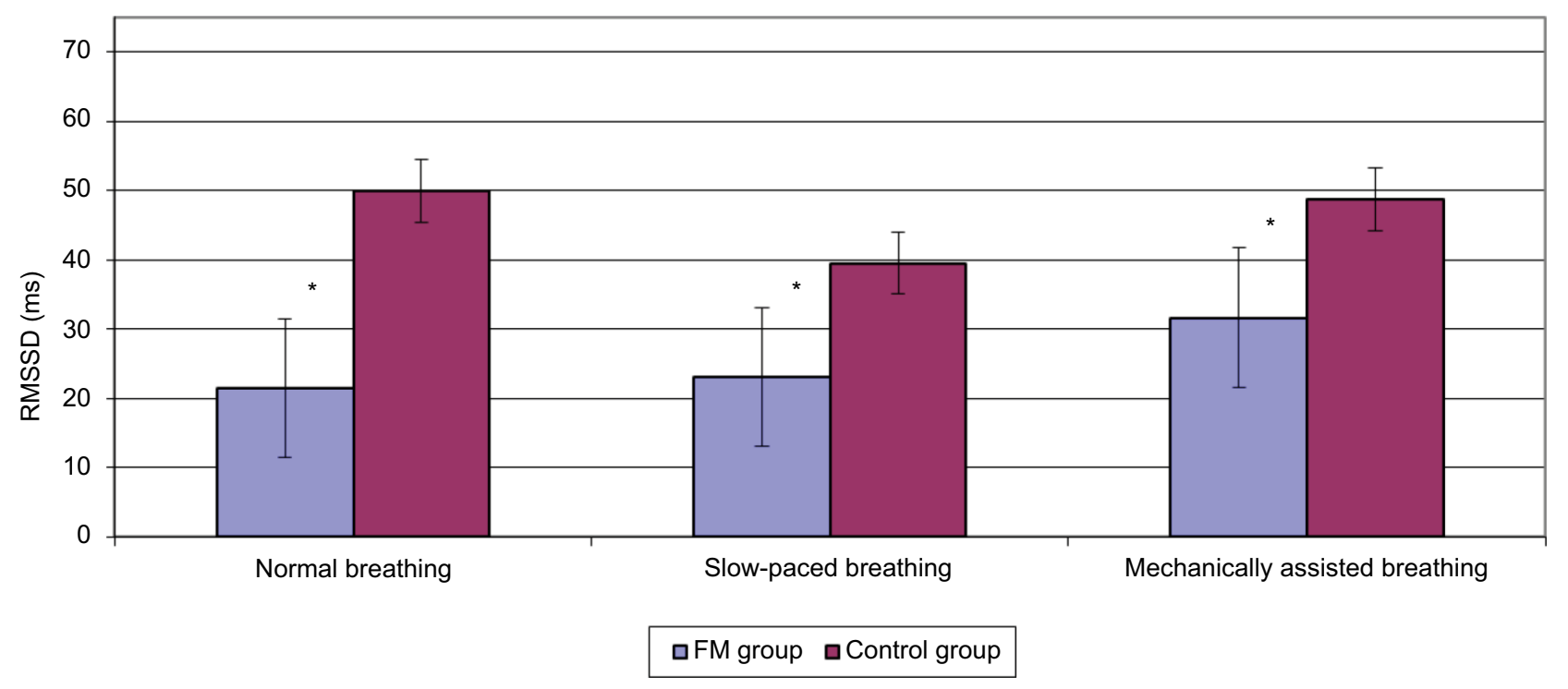

Figure 2 RMSSD function across conditions by group.

Note: ${ }^{*} p<0.05$.

Abbreviations: FM, fibromyalgia; RMSSD, root mean square of the standard deviation of normal-to-normal intervals.

even though RR was not significantly different between the two conditions. In contrast, these measures were not significantly changed in the control group from one breathing condition to the next. These results indicate a unique increase in PSNS activity and potentially a decrease in SNS activity among FM patients undergoing mechanically assisted breathing compared to slow-paced breathing.

These data raise several interesting questions. Despite similar RRs between the slow-paced and mechanically assisted breathing conditions, measures of PSNS activity among FM patients were clearly improved during mechanically assisted breathing. This suggests that simply decreasing $\mathrm{RR}$ alone does not alter autonomic activity or explain the efficacy of slow-paced breathing for FM patients. Mechanically assisted breathing may have triggered alterations in higher level autonomic centers that change the balance between SNS and PSNS activity, which were not replicated with slowpaced breathing. Although the spinal cord and peripheral autonomic ganglia play an important role in short-term ANS activity, supraspinal structures such as the hypothalamus and limbic system are crucial for long-term and anticipatory ANS regulation. ${ }^{35}$ There is overlap between the central respiratory and autonomic centers related to the connection between the hypothalamus and medullary respiratory nuclei. ${ }^{16}$

One potential explanation for our findings is that voluntary slow-paced breathing is mediated via cortical pathways, whereas mechanically assisted breathing activates hypothalamic pathways that are integrated with autonomic regulation. It is important to note that, unlike FM patients undergoing a multidisciplinary treatment program, our subjects had no prior experience with slow-paced breathing. It is reasonable to believe that, with training, slow-paced breathing could activate the involuntary hypothalamic pathways that cause long-term adaptations in autonomic regulation. More research will be needed to define these supraspinal pathways and how they are altered by controlled breathing techniques.

Given the fact that sympathetic activity and HRV indices are correlated with pain intensity in FM patients, it is important to develop treatments that improve autonomic regulation. However, prior research disagrees on exactly how altered ANS activity causes exacerbation of pain symptoms. Findings of both sympathetic hyperactivity and hypo-reactivity suggest that the physiologic relationship between SNS activity and chronic pain may be more complex than we once thought. ${ }^{21,27}$ In addition, a few studies have actually shown normal muscle sympathetic nerve activity and normal autonomic reactivity tests in patients with FM, challenging the idea that SNS overactivity is a primary physiologic mechanism in FM..$^{15,22}$ An alternative theory is that deconditioning results in peripheral ANS alterations (high SNS tone and low PSNS tone) that cause regional ischemia, which leads to widespread pain. ${ }^{21}$ This could explain why exercise is such an effective therapy for FM patients and others with chronic pain syndrome. In reality, there is likely a complex interplay between deconditioning, peripheral ANS activity, and central autonomic drive that is responsible for the varied symptoms seen in FM patients.

There are several limitations of this study that narrow the conclusions that can be drawn. This is a relatively small 
study with a total of 34 participants; hence, the results may not apply to all patients with FM, especially given the varied clinical presentations seen with this disease and the lack of understanding regarding its pathophysiology. The study design did not allow for randomization of breathing conditions, thus there is a potential for an order effect that may have influenced the data. In addition, it is unclear whether some healthy controls could actually have abnormalities in ANS activity amenable to controlled breathing techniques, depending on their comorbidities and risk factors. ${ }^{49}$ We did not directly assess sympathetic activity. Our assessments using SBRS and RMSSD are generally considered measures of vagal tone or the lack thereof when low. We did not collect data on $\mathrm{CO}_{2}$ levels or operational lung volume (stretch), both of which may have contributed to our findings. ${ }^{31,42}$

The questions raised by these data will require further study to elucidate the complex interactions between ANS activity and chronic pain, in addition to the role that controlled breathing techniques can play as part of a multidisciplinary treatment approach. Future directions should include larger studies with patients experienced at slow-paced breathing to test the hypothesis that there is a learning and/ or entrainment component responsible for the differences in ANS activity seen between slow-paced and mechanically assisted breathing. Incorporating functional MRI could also shed light on how central autonomic processes are altered with these techniques. By further understanding the physiology behind the symptoms of FM and the treatments shown to be efficacious, we will be able to better refine and individualize these therapies in the future.

\section{Acknowledgments}

Support was provided by the Mayo Clinic CTSA: RR-024150. Dr Joyner is the Frank R and Shari Caywood Professor of Anesthesiology at Mayo Clinic College of Medicine. The authors would like to thank Pam Engrav, Christopher Johnson, and Heather Tonyan for their invaluable assistance with this study.

\section{Disclosure}

The authors report no conflicts of interest in this work.

\section{References}

1. [No authors listed]. Heart rate variability: standards of measurement, physiological interpretation and clinical use. Task Force of the European Society of Cardiology and the North American Society of Pacing and Electrophysiology. Circulation. 1996;93(5):1043-1065.

2. Alok R, Das SK, Agarwal GG, Salwahan L, Srivastava R. Relationship of severity of depression, anxiety and stress with severity of fibromyalgia. Clin Exp Rheumatol. 2011;29(6 Suppl 69):S70-S72.
3. Arnold LM, Clauw DJ, Dunegan LJ, Turk DC; FibroCollaborative. A framework for fibromyalgia management for primary care providers. Mayo Clin Proc. 2012;87(5):488-496.

4. Arnold LM, Clauw DJ, McCarberg BH; FibroCollaborative. Improving the recognition and diagnosis of fibromyalgia. Mayo Clin Proc. 2011; 86(5):457-464.

5. Berntson GG, Bigger JT Jr, Eckberg DL, et al. Heart rate variability: origins, methods, and interpretive caveats. Psychophysiology. 1997;34(6):623-648.

6. Berntson GG, Cacioppo JT, Grossman P. Whither vagal tone. Biol Psychol. 2007;74(2):295-300.

7. Bertinieri G, di Rienzo M, Cavallazzi A, Ferrari AU, Pedotti A, Mancia G. A new approach to analysis of the arterial baroreflex. J Hypertens Suppl. 1985;3(3):S79-S81.

8. Buysse DJ, Reynolds CF 3rd, Monk TH, Berman SR, Kupfer DJ. The Pittsburgh Sleep Quality Index: a new instrument for psychiatric practice and research. Psychiatry Res. 1989;28(2):193-213.

9. Chobanian AV, Bakris GL, Black HR, et al. The Seventh Report of the Joint National Committee on Prevention, Detection, Evaluation, and Treatment of High Blood Pressure: the JNC 7 report. JAMA. 2003; 289(19):2560-2572.

10. Choy EH, Mease PJ. Key symptom domains to be assessed in fibromyalgia (outcome measures in rheumatoid arthritis clinical trials). Rheum Dis Clin North Am. 2009;35(2):329-337.

11. Clauw DJ. Fibromyalgia: a clinical review. JAMA. 2014;311(15): $1547-1555$.

12. Clauw DJ. Fibromyalgia and related conditions. Mayo Clin Proc. 2015;90(5):680-692.

13. Collins SL, Moore RA, McQuay HJ. The visual analogue pain intensity scale: what is moderate pain in millimetres? Pain. 1997;72(1-2):95-97.

14. Edwards RR, Bingham CO, Bathon J, Haythornthwaite JA. Catastrophizing and pain in arthritis, fibromyalgia, and other rheumatic diseases. Arthritis Rheum. 2006;55(2):325-332.

15. Elam M, Johansson G, Wallin BG. Do patients with primary fibromyalgia have an altered muscle sympathetic nerve activity? Pain. 1992;48(3):371-375.

16. Fortuna MG, Stornetta RL, West GH, Guyenet PG. Activation of the retrotrapezoid nucleus by posterior hypothalamic stimulation. J Physiol. 2009;587(Pt 21):5121-5138.

17. Garcia-Gonzalez MA, Vazquez-Seisdedos C, Pallas-Areny R. Variations in breathing patterns increase low frequency contents in HRV spectra. Physiol Meas. 2000;21(3):417-423.

18. Hassett AL, Radvanski DC, Vaschillo EG, et al. A pilot study of the efficacy of heart rate variability (HRV) biofeedback in patients with fibromyalgia. Appl Psychophysiol Biofeedback. 2007;32(1):1-10.

19. Jovanov E. On spectral analysis of heart rate variability during very slow yogic breathing. Conf Proc IEEE Eng Med Biol Soc. 2005;3:2467-2470.

20. Kang JH, Kim JK, Hong SH, Lee CH, Choi BY. Heart rate variability for quantification of autonomic dysfunction in fibromyalgia. Ann Rehabil Med. 2016;40(2):301-309.

21. Kulshreshtha P, Deepak KK. Autonomic nervous system profile in fibromyalgia patients and its modulation by exercise: a mini review. Clin Physiol Funct Imaging. 2013;33(2):83-91.

22. Kulshreshtha P, Gupta R, Yadav RK, Bijlani RL, Deepak KK. A comprehensive study of autonomic dysfunction in the fibromyalgia patients. Clin Auton Res. 2012;22(3):117-122.

23. Landen $\mathrm{M}$, Wennerblom $\mathrm{B}$, Tygesen $\mathrm{H}$, et al. Heart rate variability in premenstrual dysphoric disorder. Psychoneuroendocrinology. 2004; 29(6):733-740.

24. Lehrer PM, Gevirtz R. Heart rate variability biofeedback: how and why does it work? Front Psychol. 2014;5:756.

25. Lehrer PM, Vaschillo E, Vaschillo B. Resonant frequency biofeedback training to increase cardiac variability: rationale and manual for training. Appl Psychophysiol Biofeedback. 2000;25(3):177-191.

26. Lehrer PM, Vaschillo E, Vaschillo B, et al. Heart rate variability biofeedback increases baroreflex gain and peak expiratory flow. Psychosom Med. 2003;65(5):796-805. 
27. Martinez-Martinez LA, Mora T, Vargas A, Fuentes-Iniestra M, Martinez-Lavin M. Sympathetic nervous system dysfunction in fibromyalgia, chronic fatigue syndrome, irritable bowel syndrome, and interstitial cystitis: a review of case-control studies. J Clin Rheumatol. 2014;20(3):146-150.

28. McCracken LM, Gross RT, Aikens J, Carnrike CL Jr. The assessment of anxiety and fear in persons with chronic pain: a comparison of instruments. Behav Res Ther. 1996;34(11-12):927-933.

29. Meeus M, Goubert D, De Backer F, et al. Heart rate variability in patients with fibromyalgia and patients with chronic fatigue syndrome: a systematic review. Semin Arthritis Rheum. 2013;43(2): 279-287.

30. Mozer M, Fadel P, Johnson C, et al. Acute slow-paced breathing increases periods of sympathetic nervous system quiescence (1170.12). FASEB J. 2014;28(1).

31. Poyhonen M, Syvaoja S, Hartikainen J, Ruokonen E, Takala J. The effect of carbon dioxide, respiratory rate and tidal volume on human heart rate variability. Acta Anaesthesiol Scand. 2004;48(1):93-101.

32. Radloff LS. The CES-D scale: a self-report depression scale for research in the general population. Appl Psychol Meas. 1977;1(3):385-401.

33. Radloff LS, Locke BZ. The community mental health assessment survey and the CES-D scale. In: Weissman MM, Ross CE, editor. Community Surveys. Piscataway, NJ: Rutgers University Press; 1977:177-189.

34. Ritz T. Studying noninvasive indices of vagal control: the need for respiratory control and the problem of target specificity. Biol Psychol. 2009;80(2):158-168.

35. Salman IM. Major autonomic neuroregulatory pathways underlying short- and long-term control of cardiovascular function. Curr Hypertens Rep. 2016;18(3):18.

36. Sato N, Miyake S, Akatsu J, Kumashiro M. Power spectral analysis of heart rate variability in healthy young women during the normal menstrual cycle. Psychosom Med. 1995;57(4):331-335.

37. Schmidt JE, Joyner MJ, Carlson CR, Hooten WM. Cardiac autonomic function associated with treatment adherence after a brief intervention in patients with chronic pain. Appl Psychophysiol Biofeedback. 2013;38(3):193-201.
38. Schmidt JE, Joyner MJ, Tonyan HM, Reid KI, Hooten WM. Psychological and physiological correlates of a brief intervention to enhance self-regulation in patients with fibromyalgia. J Musculoskelet Pain. 2012;20(3):211-221.

39. Siepmann M, Aykac V, Unterdorfer J, Petrowski K, Mueck-Weymann M. A pilot study on the effects of heart rate variability biofeedback in patients with depression and in healthy subjects. Appl Psychophysiol Biofeedback. 2008;33(4):195-201.

40. Song HS, Lehrer PM. The effects of specific respiratory rates on heart rate and heart rate variability. Appl Psychophysiol Biofeedback. 2003;28(1):13-23.

41. Spielberger CD, Gorsuch RL, Lushene RE. Manual for the Stat-Trait Anxiety Inventory (Form Y). Palo Alto, CA: Consulting Psychologists Press; 1983.

42. Stauss HM. Heart rate variability. Am J Physiol Regul Integr Comp Physiol. 2003;285(5):R927-R931.

43. Stein KD, Martin SC, Hann DM, Jacobsen PB. A multidimensional measure of fatigue for use with cancer patients. Cancer Pract. 1998;6(3):143-152.

44. Tracy LM, Ioannou L, Baker KS, Gibson SJ, Georgiou-Karistianis N, Giummarra MJ. Meta-analytic evidence for decreased heart rate variability in chronic pain implicating parasympathetic nervous system dysregulation. Pain. 2016;157(1):7-29.

45. Weissman MM, Sholomskas D, Pottenger M, Prusoff BA, Locke BZ. Assessing depressive symptoms in five psychiatric populations: a validation study. Am J Epidemiol. 1977;106(3):203-214.

46. Wolfe F, Clauw DJ, Fitzcharles MA, et al. Fibromyalgia criteria and severity scales for clinical and epidemiological studies: a modification of the ACR preliminary diagnostic criteria for fibromyalgia. J Rheumatol. 2011;38(6):1113-1122.

47. Zamuner AR, Barbic F, Dipaola F, et al. Relationship between sympathetic activity and pain intensity in fibromyalgia. Clin Exp Rheumatol. 2015;33(1 Suppl 88):S53-S57.

48. Zamuner AR, Forti M, Andrade CP, Avila MA, da Silva E. Respiratory sinus arrhythmia and its association with pain in women with fibromyalgia syndrome. Pain Pract. 2016;16(6):704-711.

49. Zautra AJ, Fasman R, Davis MC, Craig AD. The effects of slow breathing on affective responses to pain stimuli: an experimental study. Pain. 2010;149(1):12-18.
Journal of Pain Research

\section{Publish your work in this journal}

The Journal of Pain Research is an international, peer reviewed, open access, online journal that welcomes laboratory and clinical findings in the fields of pain research and the prevention and management of pain. Original research, reviews, symposium reports, hypothesis formation and commentaries are all considered for publication.

\section{Dovepress}

The manuscript management system is completely online and includes a very quick and fair peer-review system, which is all easy to use. Visit http://www.dovepress.com/testimonials.php to read real quotes from published authors. 